\title{
Long-term X-ray variability in blazars and its multi- waveband context
}

\author{
Alan P. Marscher \\ Institute for Astrophysical Research, Boston University \\ 725 Commonwealth Ave., Boston, MA 02215, USA \\ E-mail: marscher@bu.edu
}

\begin{abstract}
The accumulation of well-sampled X-ray, optical, and radio light curves of blazars and coredominated radio galaxies allows analysis of the long-term variability properties and crossfrequency correlations. Armed with this rich database, we are starting to be able to determine where in the jet the nonthermal flares take place. We find that these can occur in at least two locations: in the core seen on millimeter-wave VLBI images and in a region with a helical magnetic field well upstream of the core. The latter is consistent with the jet acceleration and collimation zone according to the magnetohydrodynamical models favored by most theorists. In this picture, the jet of a blazar, which points within several degrees of the line of sight, produces the bulk of its radiation near the end of this zone and in standing shocks or other compressions downstream. The outbursts and superluminal radio knots are connected with events in the central engine signalled by dips in the X-ray flux. However, a model detailing how the accretion-disk/black-hole system couples with the jet still needs to be developed.
\end{abstract}

Workshop on Blazar Variability across the Electromagnetic Spectrum

Palaiseau, France

April $22^{\text {nd }}-25^{\text {th }} 2008$ 


\section{Introduction}

Blazars are fascinating objects, but at the same time frustrating. Their prominent variations of nonthermal emission across the electromagnetic specrum provide a rich dataset for understanding the most energetic processes that occur in nature. But this same dataset is filled with complexities that defy attempts to interpret the physical phenomena behind the variations. The history of research on blazars is filled with attempts to unlock their secrets by observing selected objects intensively over periods ranging from days to weeks. Such "campaigns" have indeed provided glimpses of insight into the physics of flares and other events. However, a more complete picture requires nearly as intensive monitoring over longer terms. This involves wellsampled light curves at many wavebands, as well as imaging with very long baseline interferometry (VLBI) at centimeter and millimeter wavelengths, combined with repeated observations of polarization at optical-infrared wavelengths.

Long-term monitoring has been ongoing for decades at radio and optical observatories, although well-sampled, long-term optical light curves have been restricted to only a few objects. The situation has been less favorable at X-ray and $\gamma$-ray energies, at which light curves generally have been poorly sampled. Since its launch in 1995, the Rossi X-ray Timing Explorer (RXTE) has solved this problem at medium X-ray energies (between 2.4 and either 10 or 20 $\mathrm{keV}$, depending on the flux and flatness of the spectrum). Using RXTE, we have been able to accumulate well-sampled, long-term light curves of a number of active galactic nuclei (AGN) over many years. My collaborators and I have been doing so for four blazars - 3C 273, 3C 279, PKS 1510-089, and BL Lac - and two radio galaxies with blazar-like behavior at radio to farinfrared wavelengths - 3C 111 and 3C 120. Here I report on what we have learned thus far about the nature of relativistic jets in active galactic nuclei from these multi-waveband monitoring studies.

\section{Link between relativistic jets and outbursts of radiation in blazars}

Thanks to radio interferometry, we have understood since the early 1980 s that the nonthermal emission from radio to $\gamma$-ray frequencies arises from jets of magnetized plasma that flow out from the nucleus at relativistic velocities (see Figure 1). In most blazars, sequences of VLBI images feature bright knots of emission that move away from a compact, essentially stationary "core" at superluminal apparents speeds. (Note that the core seen on VLBI images is displaced from the black hole by a distance of order $1 \mathrm{pc}$ or more $[14,15]$.) It is natural to hypothesize that these superluminal "blobs" are related to the outbursts of radiation for which blazars are famous. However, since we can only attain the sub-milliarcsecond angular resolution needed to find and follow the blobs at radio frequencies, and since the jet becomes opaque to radio emission at or a bit upstream of the core, it is not so straightforward to demonstrate a link between the blobs and flares at optical, X-ray, and $\gamma$-ray frequencies. 

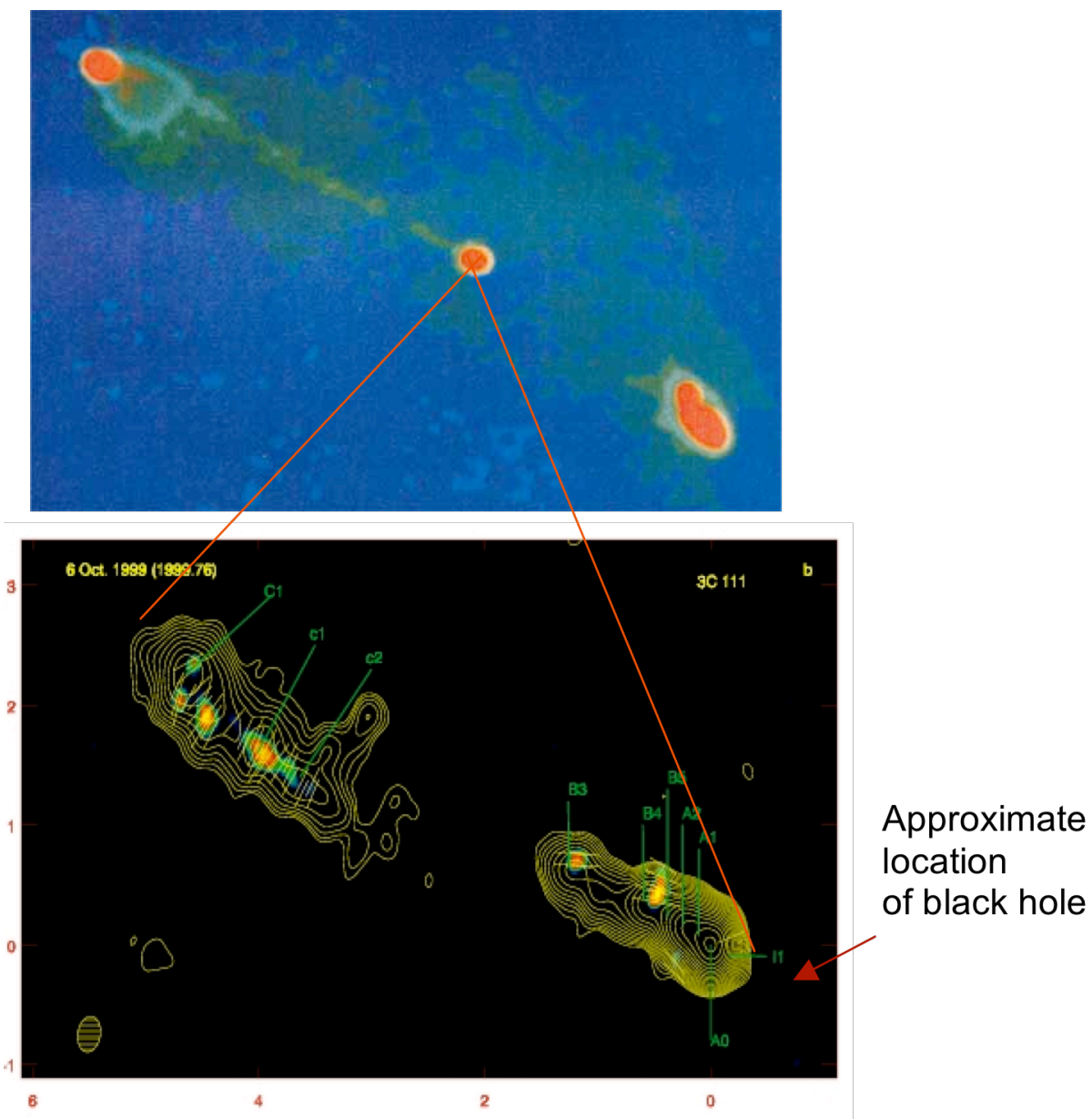

Relative R.A. (milliarcseconds)

Figure 1. Images of the radio galaxy 3C 111. Top: Very Large Array (VLA) image from the image archive of the National Radio Astronomy Observatory. The distance from the nucleus (center) to the northeast hotsplot is about 2 arcminutes. Bottom: Image of the nuclear region at $43 \mathrm{GHz}$ obtained with the Very Long Baseline Array (VLBA) [from 9]. Contours, in ratios of a factor of 2, corresond to total intensity, while false colors indicate the polarized intensity. The lengths of the yellow sticks indicate fractional polarization and the orientations correspond to the electric field vector directions. The core is marked as component A0, while the B and C components are knots (or "blobs") moving away from the core at superluminal speeds.

The motions of the blobs allow us to determine where they were located at some time in the past, under the assumption that the apparent velocities are constant. If we can associate a flare at high frequencies with a particular blob, then we can determine where the flare occurred relative to the core. But that "if" is a major issue. A typical blazar produces 1-3 bright superluminal knots per year and exhibits variations in flux that have the power spectral density of red noise. This means that there are many flares with various amplitudes and time scales, so associating any particular one with a given blob is problematic.

There are two solutions to this dilemma. One is to accumulate enough data that a crosscorrelation analysis can be performed with a low probability of spurious connections between flares at different wavebands. If the appearance of a superluminal blob on $43 \mathrm{GHz}$ Very Long 
Baseline Array (VLBA) images (see Fig. 2) is accompanied by a flare at $37 \mathrm{GHz}$ (at which the Metsähovi Radio Observatory monitors the fluxes of blazars), as is usually the case [20], we can cross-correlate the $37 \mathrm{GHz}$, optical, and X-ray light curves to determine whether the expected connection between superluminal ejections and high-frequency flares actually exists. If we can establish that this is the case, then we can analyze individual events to determine the time delays between peaks in the high-frequency light curves and times when the corresponding blob was coincident with the core. We can then determine the locations of the high-frequency flares relative to the core.

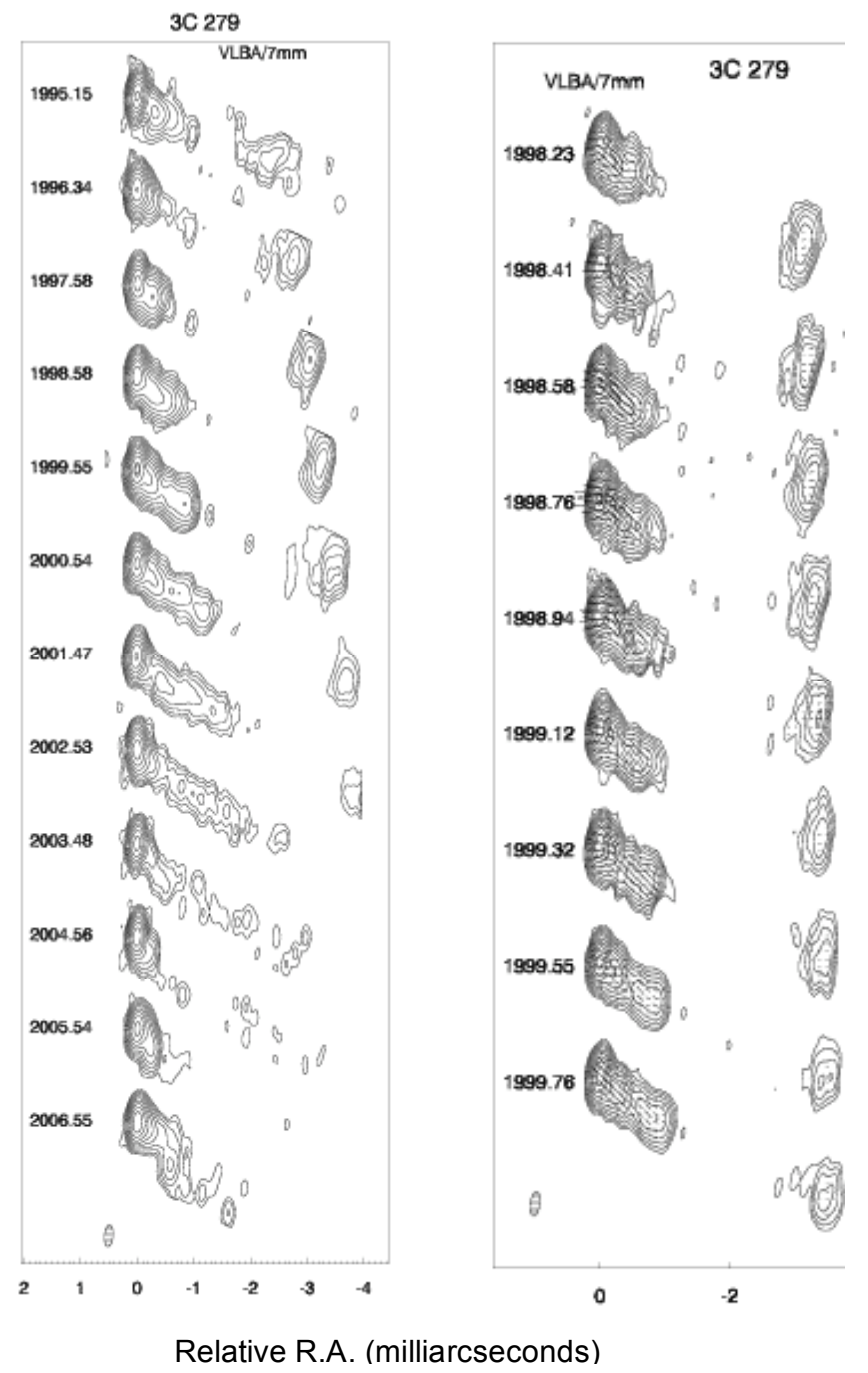

Figure 2. Sequences of VLBA images of the quasar 3C 279 at a frequency of $43 \mathrm{GHz}$. The core is the bright feature on the northeastern end. Left: One image per year; notice how the direction of the jet near the core changes. Right: Bimonthly images during a 1.5-year interval. In both sequences, the apparent superluminal motion of bright knots is apparent. From [4] and [9]

The other solution is to use polarization to connect a particular blob with a flare at optical wavelengths. In many cases, the polarization electric-vector position angle (EVPA) is different from that of other bright features in the jet. If this same, unique EVPA occurs in the optical 
polarization during a flare, we can conclude that the blob is very likely the primary source of the optical emission. Once this association is established, we can use correlations and time lags between the high-energy and optical (or radio) light curves to determine where the X-ray and $\gamma$ ray emission arises in the jet.

\section{Long-term multi-waveband light curves of $3 \mathrm{C} 279$}

Figure 3 presents the long-term light curve of the blazar 3C 279 at X-ray, optical, and radio frequencies. Marked along the bottom of the top panel are the times when new superluminal knots were coincident with the core, the presumably stationary bright feature at the upstream end of the jet on $43 \mathrm{GHz}$ VLBA images (see Fig. 2).

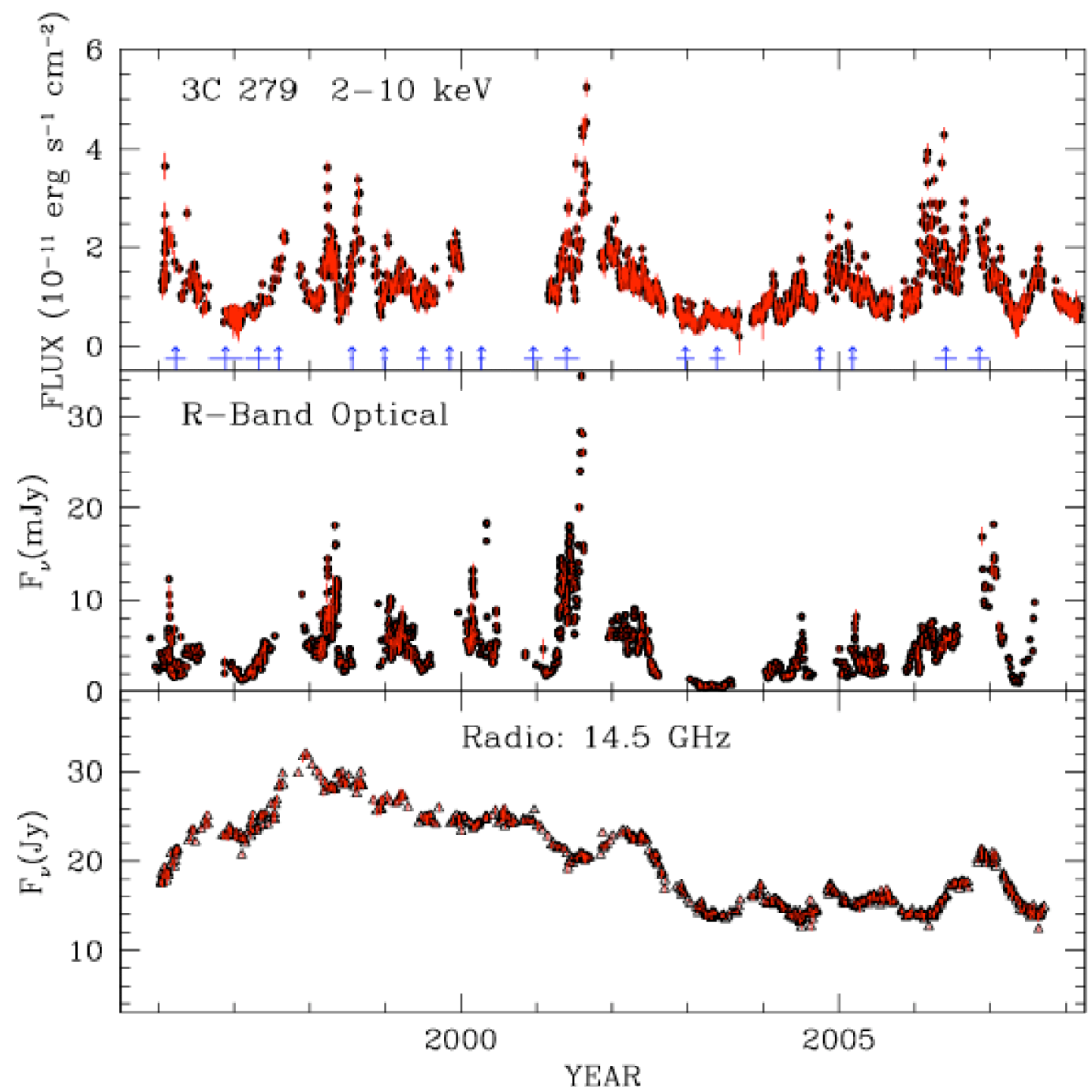

Figure 3. Light curves of the quasar 3C 279 at three wavebands. Along the bottom of the top panel, the times of superluminal ejections are marked with upward arrows, with the horizontal bar indicating the uncertainty in the date. Data are from [4]. 
The emission at all three wavebands is nonthermal: synchrotron radiation at radio to optical frequencies and inverse Compton scattering at X-ray energies. Thermal radiation from the accretion disk is prominent at higher optical and ultraviolet frequencies (see the spectral energy distribution shown in Fig. 4). The seed photons for the X-ray emission are probably the same synchrotron photons that the scattering electrons produce in the jet, i.e., the emission

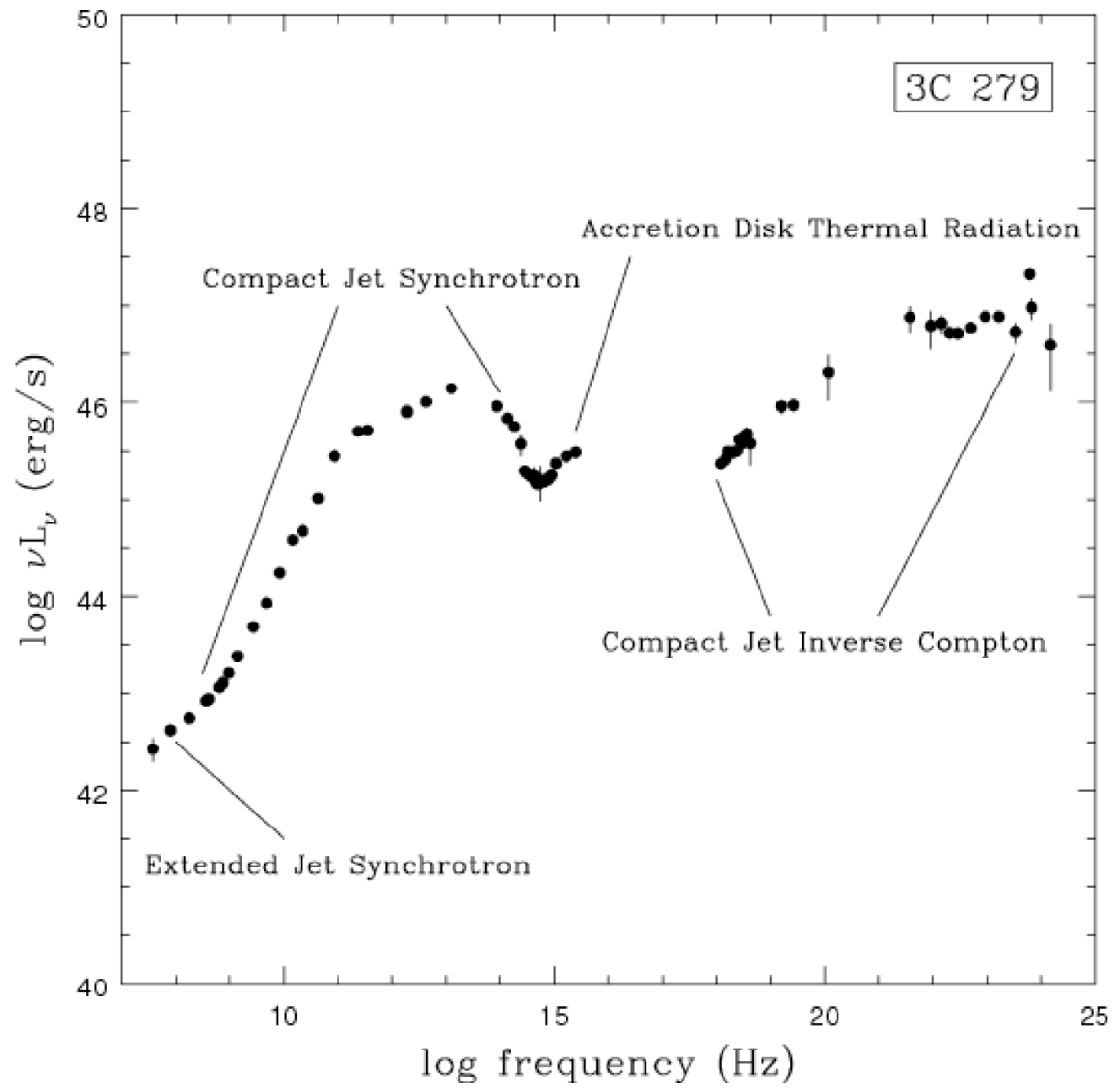

Figure 4. Spectral energy distribution of $3 \mathrm{C} 279$, from data by the author and collaborators and from the NASA Extragalactic Database. The primary process and location of the emission at different frequency ranges are indicated.

process is synchrotron self-Compton scattering. There is roughly equal contribution from optical photons scattered by relatively low-energy electrons, IR photons scattered by intermediate-energy electrons, and mm-wave photons scattered by high-energy electrons [19]. If there are gradients in electron energy, as may occur if particles are accelerated mainly at shock fronts [18], the optical emission, generated solely by the highest-energy electrons, will occur over a smaller volume than does either the IR/mm-wave or the X-ray emission.

One can readily see through inspection of Figure 3 that there is a general correspondence between the optical and X-ray light curves. There are a number of events during which both wavebands varied in a similar way: the prominent outburst in 2001, the year-long low flux level 
in 2003, and the deep, sharp minimum at 2007.3. The $14.5 \mathrm{GHz}$ light curve shows some of the same events, but time delayed and smoothed. This indicates that the high-frequency action occurs upstream of the portion of the jet that is transparent at $14.5 \mathrm{GHz}$. In addition, the lifetimes of electrons emitting at $14.5 \mathrm{GHz}$ are longer than at higher frequencies, so the radio flux at any given time contains a superposition of many knots.

A careful correlation study [4] provides a more quantitative analysis of the relationship of the emission at the three wavebands. This involves determination of the power spectral density (PSD) of the variations from the raw PSD and the time sampling via comparison of simulated light curves with actual data. The PSD at each waveband corresponds to red noise that can be fit by a single power law of order -2 . The optical slope is flatter than the X-ray and radio slopes. This indicates that the optical emission is more highly variable on the shortest time-scales to which the observations are sensitive (a few days) than is the case for the radio and X-ray flux, in agreement with the notion that a typical optically emitting electron has a higher energy and shorter radiative lifetime than does the average electron responsible for either the radio or X-ray emission. This is corroborated by the flare profiles: the $\mathrm{X}$-ray profiles tend to be broader than is the case for contemporaneous optical flares. Despite this, in 6 out of 13 flares the X-ray peak is earlier than the optical. This implies that, in nearly half of the outbursts, it takes extra time to accelerate electrons up to their highest energies. Böttcher et al. [2] arrived at a similar conclusion after studying 3C 279 over a much more limited time interval.

The time delay between the peaks of X-ray and optical flares in 3C 279 changes on a timescale of years. For some period the X-rays lead by $\sim 20$ days, then the variations are essentially simultaneous, and then the optical leads by $\sim 20$ days. The most striking outburst, in 2001, followed a switch from X-ray leading to optical leading. Another long-term trend is that the flux at all three wavebands was lowest when the jet swung by $\sim 30^{\circ}$ to the south in 2002-03 (see Fig. 2 ), just after the big outburst. The formal correlation indicates that changes in the position angle lead those in the X-ray flux by $80 \pm 150$ days. This implies that the swing in jet direction mainly modulates the overall flux level by gradually changing the Doppler factor instead of stimulating a sharp event in the light curve.

By integrating the flux across the flare profile, we can compare the total radiated energy of X-ray and optical flares. Chatterjee et al. [4] find that when the optical exceeds the X-ray output, the time delay tends to be longer. This is consistent with such flares originating downstream in the jet where the emission region is less compact. Synchrotron self-Compton scattering is therefore less efficient at producing X-rays than in the more compact plasma farther upstream. In addition, time lags from light-travel delays (time for the seed photons to cross the source) or gradients in electron energy behind a shock front [18] are longer when the emitting region is larger.

The relation between the high-frequency flares and the emergence of new superluminal radio knots is rather tricky to establish, since there are many events of both types and the time delays seem to be variable. In addition, the most prominent outburst, in 2001, was followed by a major brightening in the $43 \mathrm{GHz}$ core on the VLBA images, but no particularly bright knot emerged. (The same was true of the 2005 outburst in 3 C 454.3.) The simplest explanation is that the disturbance was quenched by severe radiative energy losses of the electrons, probably 
corresponding to a very high (but unobserved owing to absence of a $\gamma$-ray telescope) flux of $\gamma$ rays produced via inverse Compton scattering. Nevertheless, we can use flares in the core at 43 $\mathrm{GHz}$ as a proxy for the birth of a superluminal knot [20]. Chatterjee et al. [4] find that the X-ray flux is weakly correlated with the core flux, with a time lag of $130_{-45}^{+70}$ days. Correlation of the X-ray and $37 \mathrm{GHz}$ (from Metsähovi Radio Observatory) light curves and comparison of the timing of $37 \mathrm{GHz}$ flares relative to superluminal ejections demonstrates that the weak Xray/core correlation is due mainly to a varying time lag. Some of the ejections, especially those following the highest amplitude flares, lag the peaks of the X-ray flares by over 100 days, while others actually precede the X-ray maxima [17].

The overall conclusion from this analysis of the long-term behavior of 3C 279 is that the optical and X-ray emission is produced at multiple locations in the jet, both near and downstream of the $43 \mathrm{GHz}$ core and well upstream of the core. The time lags observed between high-frequency and radio events can exceed 100 days. Given the apparent superluminal motion of nearly $20 c$ and an angle to the line of sight of $\sim 2^{\circ}$ [9], this translates to a distance of tens of parsecs between the most upstream site of optical and X-ray emission and the $43 \mathrm{GHz}$ core. Despite this large distance, things happen fast in 3C 279 owing to its high Doppler factor, which probably always exceeds 20 .

\section{The double flare of BL Lac in Late 2005: where the action is}

The power of the technique described in $\$ 2$ for probing the innermost regions of blazar jets was demonstrated dramatically by observations of the late- 2005 double flare of BL Lac [16]. Figure 5 presents the multi-waveband light curves. During the first flare, seen at optical,
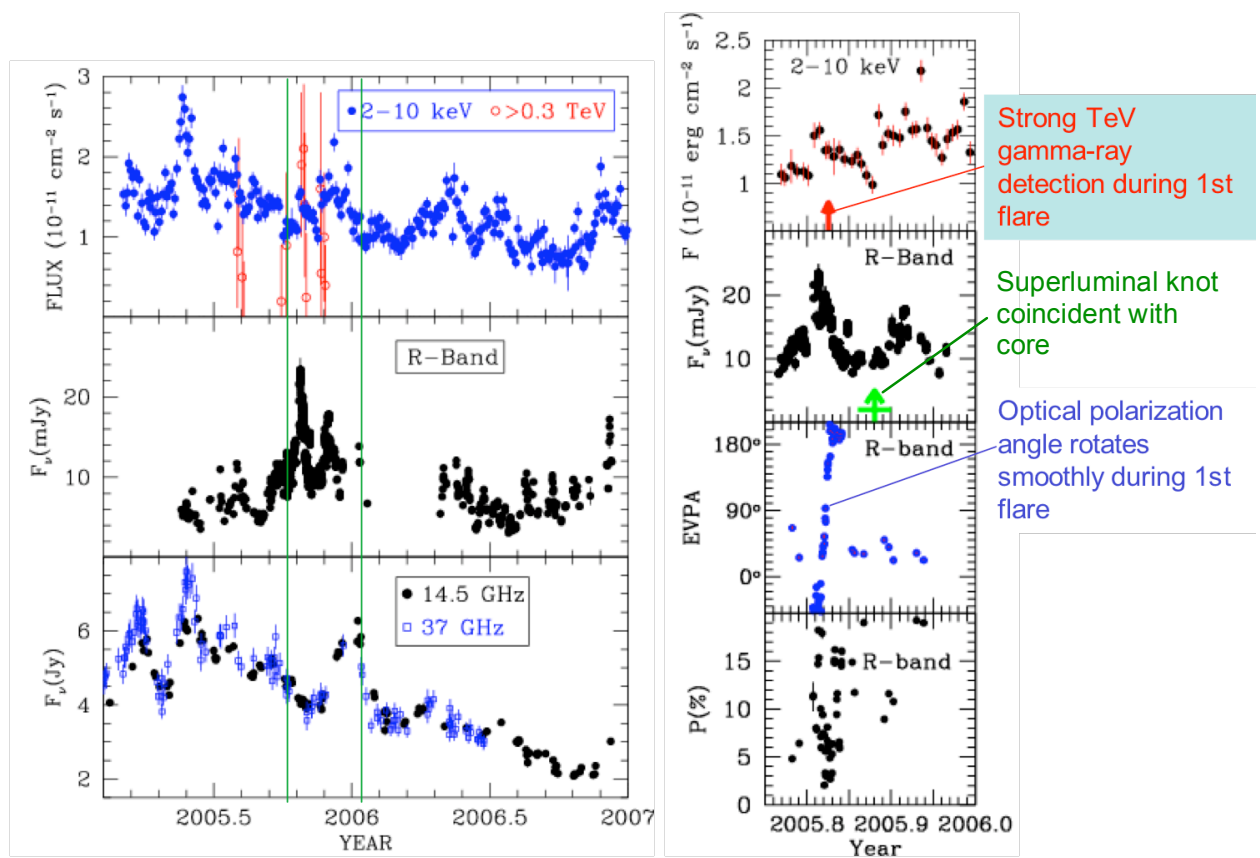

Figure 5. Multi-waveband light curves of BL Lac. Panel to right corresponds to the time interval delineated by the green vertical lines in the lefthand panel. Data for plots are from [16], except for the $>0.3 \mathrm{TeV}$ points, which are from [1]. 
$\mathrm{X}$-ray, and $\mathrm{TeV} \gamma$-ray - but not at radio - frequencies, the optical polarization vector executed a smooth rotation by over $200^{\circ}$ as the flux rose. Although such rotations of the polarization position angle $\chi$ have been observed previously in BL Lac and a few other blazars, the older data were too sparse to confirm the smoothness of the rotation. Apparent rotations can occur via random walks when the emitting plasma is actually quite turbulent [8]. But in this case, $\chi$ fluctuates randomly about the mean trend of rotation, whereas the rotation that we observe is quite smooth (see Fig. 6).

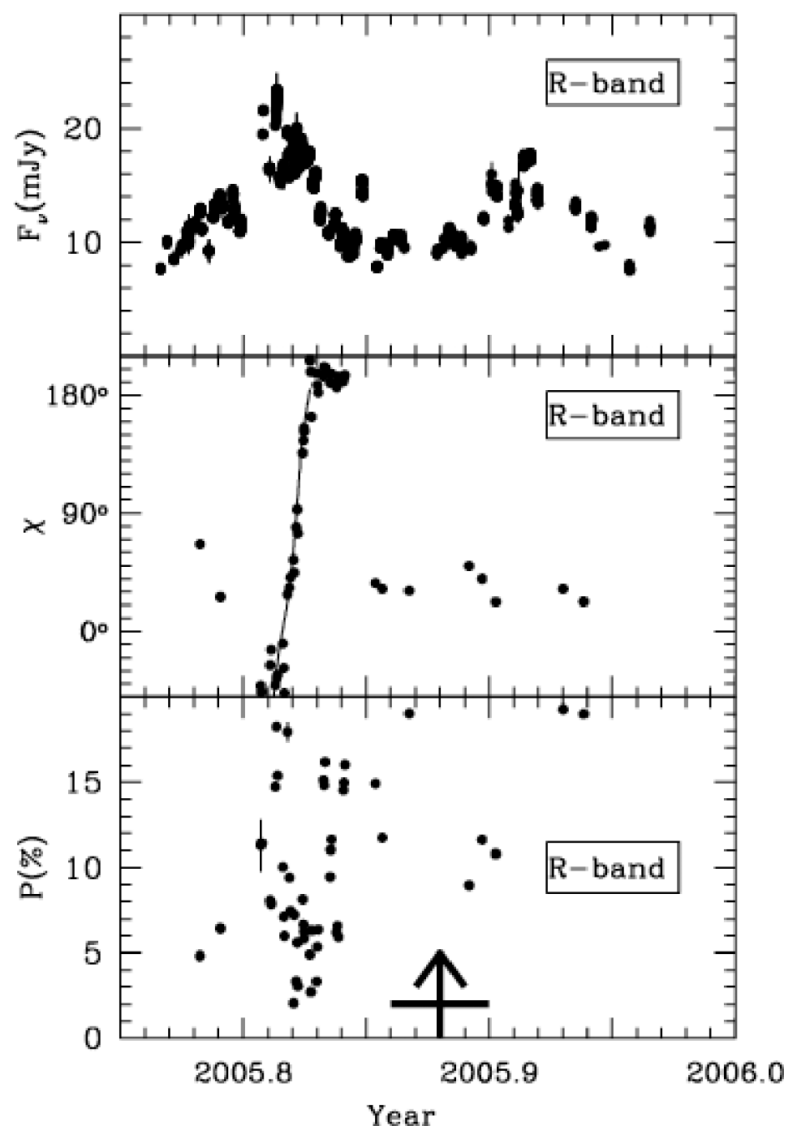

Figure 6. Optical R-band flux density (top), polarization position angle (middle), and degree of polarization (bottom) as a function of time for BL Lac in late 2005. Data are from [16]. The curve in the middle panel corresponds to the rotation of $\chi$ expected for a synchrotron-emitting disturbance following a spiral streamline that passes through a tightly wound helical magnetic field, after the effect of relativistic aberration is included.

The observed rotation of $\chi$ is the signature of the disturbance causing the flare passing through a section of the jet that contains a helical magnetic field. In order for there to be a net polarization that circulates smoothly across the full range of orientations, two conditions need to be satisfied: (1) the disturbance cannot both be uniform and extend across the entire crosssection of the jet, otherwise the net linear polarization would be persistently perpendicular to the projected direction of the jet [13]; and (2) the motion of the bright feature must spiral about the jet axis. We therefore have the picture shown in Figure 7. We cannot determine whether the 
streamline follows a thick strand of helical field lines - in which case the helix would need to be rather open - or if the field is much more tightly coiled than the streamline. If the latter case occurs, the disturbance cannot be a magnetosonic shock, which cannot propagate across the field lines.

This "toy" model, which we have derived solely from the observations, matches the theoretical scenario proposed by Vlahakis [21], which is based on numerical simulations of jets that are driven, accelerated, and collimated by magnetic forces. As is discussed in the supplemental information of [16], the circular velocity of the emission feature is consistent with

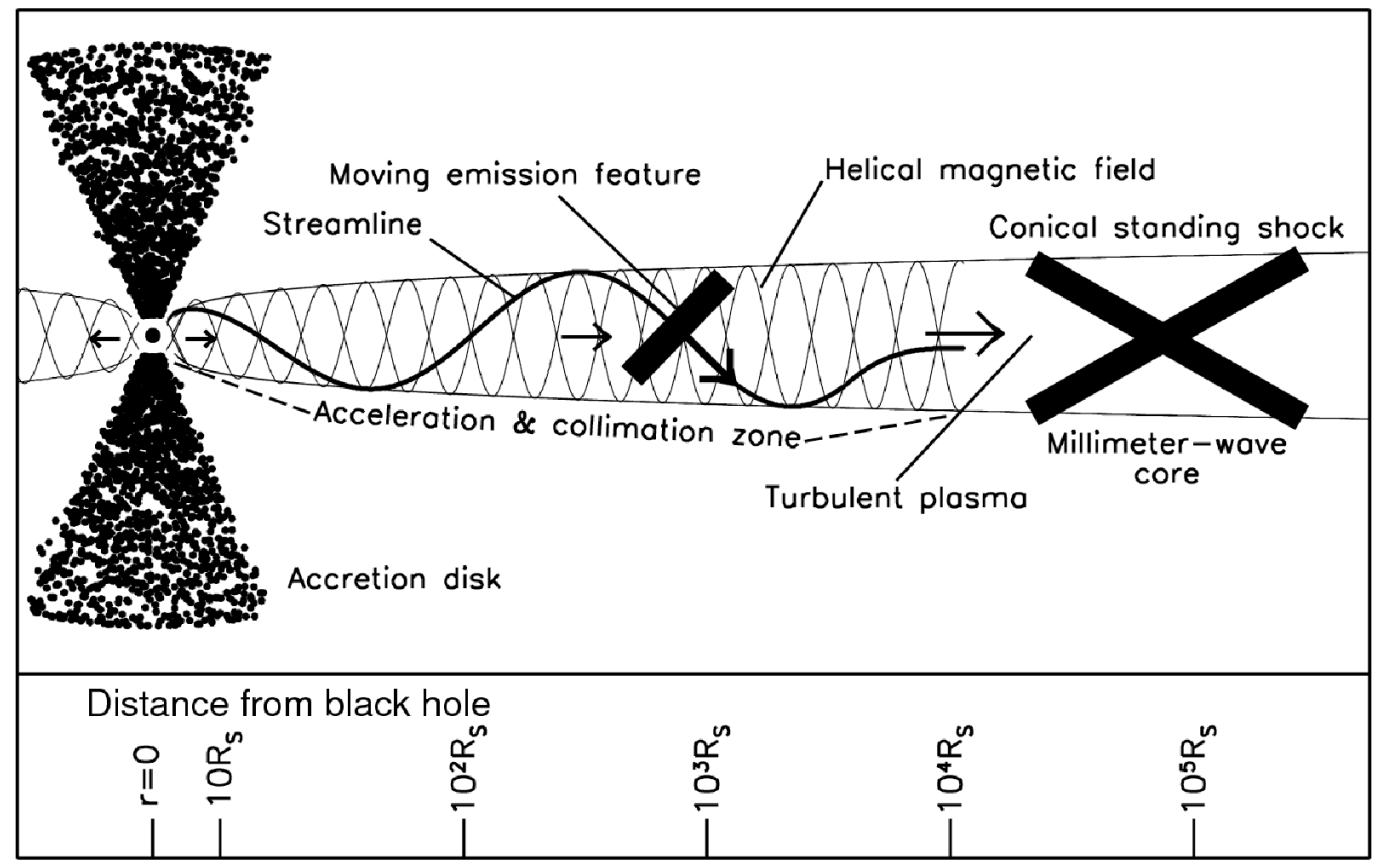

Figure 7. Model for BL Lac. The first flare in late 2005 occurred as the emission feature made its last loop in the acceleration and collimation zone. The second flare coincided with passage of the disturbance - by this time a moving shock wave - through the millimeter-wave core, which is modeled here as a standing recollimation "X-shaped" (nose-to-nose cones in three dimensions) shock system. This is a slightly modified version of Figure 3 in [16].

rigid rotation of the streamlines from either the inner accretion disk or ergosphere out to the Alfvén surface and conservation of angular momentum beyond that point.

The model has two observational requirements that extend beyond the features of the data that engendered it. The motion along the spiral streamline is aberrated (or equivalently, there are light-travel delays that must be taken into account). This is equivalent to viewing the jet at an angle of $\sim 50^{\circ}$ rather than the actual angle of $\sim 7^{\circ}$. The circular cross-section of the cone about which the streamline is wrapped therefore appears quite elliptical in our frame. Because of this, the rotation of the polarization with time follows a curve rather than a line. As we can see in Figure 6 (middle panel), this corresponds very well to the observed rotation. The other requirement is that the polarization from different parts of the cross-section should nearly cancel out from symmetry during the rotation, since the emission feature must cover a substantial fraction of the cross-section of the jet for its flux to be significant. Indeed, the mean degree of polarization is lower during the rotation $(\sim 4 \%)$ than before and after $(10-18 \%)$. The matching of 
these features of the observations with the theoretical explanations gives us confidence that the basic model of helical motion through a helical magnetic field corresponds to reality.

Since the jet of BL Lac possesses similar morphology as that of other blazars, and since the variability of its multi-waveband flux, while extreme, is also qualitatively the same as that of other highly polarized AGN, we expect that the findings discussed above should apply to many, or even all, AGN with relativistic jets. Similar well-sampled, multi-waveband timing studies including $\gamma$-ray light curves and spectra from GLAST will determine whether this is indeed the case.

\section{Components of the inner jet and the spectral energy distribution}

We now have a picture (Fig. 7) of an AGN jet that contains at least three distinct emission regions: an acceleration and collimation zone (ACZ) with helical magnetic field and spiral sreamlines, a turbulent zone, and standing conical (or, if symmetry is broken, oblique in a more complex manner) shocks. Moving shocks and other disturbances passing through these regions generate flares with distinct characteristics. We might also expect each region to produce "quiescent" emission with a particular signature.

Turbulent plasma flowing through a standing shock should produce synchrotron emission with a low degree of linear polarization that fluctuates. As a crude approximation, we can model the emission as coming from $N$ cells, each with randomly oriented magnetic field of similar strength as in other cells. The mean net polarization, which would be of order $75 \%$ if the field were uniform, is instead $\sim 0.75 N^{-1 / 2}$, while the RMS fluctuations about the mean $\sim N^{-1 / 2}$ [3]. Short-term apparent rotations in $\chi$ can occur from the random walk when the polarization is less than a few percent, but these tend to be jagged rather than smooth as was the case in BL Lac. D'Arcangelo et al. [6] have observed this behavior in the quasar 0420-014 both at optical wavelengths and in the core on $43 \mathrm{GHz}$ VLBA images during an 11-day intensive campaign. We can therefore state that the core on the $43 \mathrm{GHz}$ images was also the site of optical emission, and that the emitting plasma was turbulent. Despite the fluctuations in flux and, especially, polarization, 0420-014 was rather inactive during the campaign, hence we measured the properties of the relatively quiescent emission from the jet.

If most of the $43 \mathrm{GHz}$ and optical non-flaring radiation comes from the $43 \mathrm{GHz}$ core, we can question whether the ACZ of the jet contributes a substantial fraction of the emission at any waveband. A detailed monitoring study of the millimeter-wave and optical polarization of 15 radio-loud AGN [10; see also the paper by Jorstad in this volume] indicates that it may do so at submillimeter wavelengths. The $7 \mathrm{~mm}$ core and $3 \mathrm{~mm}$ electric-vector polarization position angles (EVPAs) tend to agree quite well (after correction for Faraday rotation) with the optical EVPA. However, the $1.3 / 0.85 \mathrm{~mm}$ EVPA generally does not, which suggests a separate component of emission. This tentative conclusion is supported by the spectral energy distributions of 3C 273 and 3C 279 displayed in Figure 8. If this component of jet emission exists, it implies that particle acceleration in the quiescent ACZ has too low efficiency to generate electrons with enough energy to radiate at optical wavelengths despite the strong magnetic field threading this zone. 


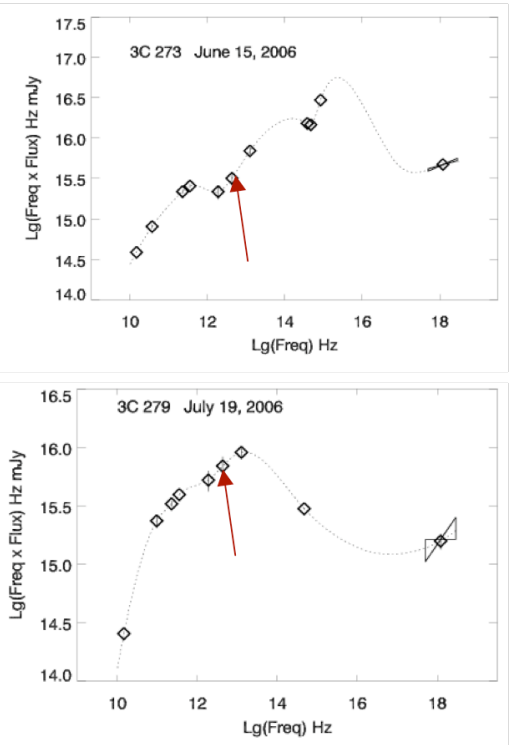

Figure 8. Spectral energy distributions of 3C 273 and 3C 279 in mid-2006. The X-ray data are from RXTE and the IR data are from the Spitzer Space Telescope, both by the author and collaborators. The red arrows indicate the presence of a far-IR component distinct from the millimeter-wave emission region. Data are from [11].

\section{Where the flares are generated}

If the jet is really launched by magnetic fields twisted by differental rotation of the plasma near the black hole, there should be a connection between events that occur in the central engine and those seen in the jet. Such a connection was claimed in the FR 1 radio galaxy $3 \mathrm{C}$ 120 [15], with the appearance of superluminal knots passing through the core on $43 \mathrm{GHz}$ VLBA images following a significant (relative to the mean level and RMS), temporary drop ("dip") in the X-ray flux, with a time lag of $\sim 60$ days. This particular object is blazar-like, i.e., jetdominated, at radio wavelengths, while most of the X-ray flux comes from the accretion disk corona system, hence it is one of the few AGN for which such a study can be carried out. The longer and better sampled dataset we have accumulated since 2002 (the original study covered the period 1997-99) confirms the dip/ejection relationship [5]. Similar, ongoing monitoring of the FR 2 radio galaxy 3C 111 suggests that it may also follow a similar pattern [14], although a longer data train is necessary to confirm the dip/ejection relationship.

Exactly what occurs in the central engine to inject faster and/or more energetic flow into the jet remains an open question even in black-hole X-ray binaries (XRBs) where there are stronger observational signatures of changes in physical conditions (see, e.g., [7]). A shock moving down the jet can be generated by a physical event that temporarily increases the velocity of the jet flow such that the relative velocity of new and old flow is supersonic. Why this should be associated with a drop in X-ray flux is unclear. Perhaps a mostly turbulent magnetic field in the accretion disk undergoes a transition to a more strongly poloidal field in the disk, which would decrease heating in the disk while promoting flow into the jet [12]. If the 


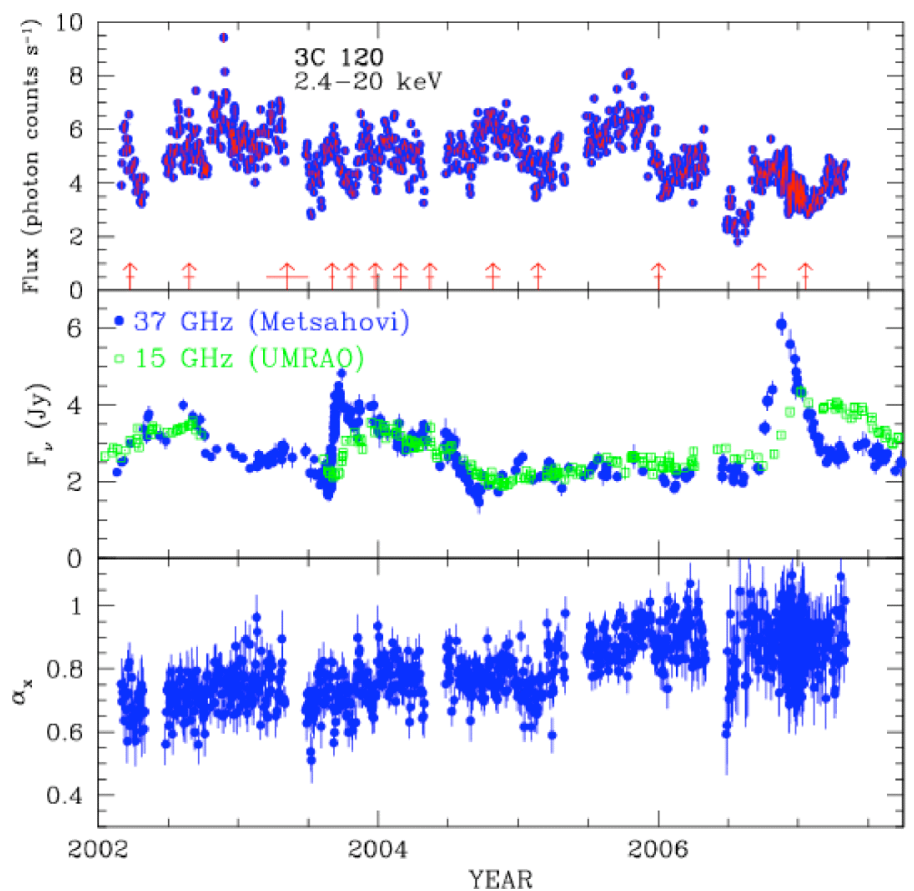

Figure 9. X-ray flux, radio flux density, and X-ray spectral index vs. time for the radio galaxy $3 \mathrm{C} 120$. The upward arrows in the top panel denote times when new superluminal knots were coincident with the core on $43 \mathrm{GHz}$ VLBA images. Data are from [5].

so-called "corona," which is responsible (via inverse Compton scattering) for essentially all of the X-ray emission from the central engine in AGN and the hard-spectrum component of the Xray emission in XRBs, is actually the base of the jet, the faster flow would (following Bernoulli's principle) decrease the density of electrons, thereby lowering the X-ray flux until the flow slows down again [14]. These models are not yet well developed yet, and more ideas and detailed theoretical simulations are needed to provide a coherent picture of how the black hole, disk, corona, and jet are coupled.

\section{Conclusion}

After decades of attempts to convert the results of monitoring programs into decisive information about the structure and physics of relativistic jets, it appears that we have learned just how extensive a dataset is needed to achieve this goal. Densely sampled imaging with VLBI at millimeter wavelengths, polarization monitoring at radio through optical (extending into the X-ray domain someday, we hope) frequencies, and total flux monitoring across the electromagnetic spectrum is the answer. It is exciting that GLAST will allow us to extend our program into the hard $\gamma$-ray domain with light curves containing flux points roughly every day for the brighter blazars. This is tempered by the unfortunate decision to shut off RXTE in early 2009 and the threat to do the same to the VLBA two years later. As we have found with 3C 279, many years of monitoring are required to sample the range of correlative behavior that occurs in a given object. We can expect that we will need to carry out such intensive, long-term, multiwaveband programs for tens of blazars before we gain a sense of all their modes of variation 
and of the multiple physical mutations to which jets are susceptible. In order to do so, we need to save the VLBA and to replace the capabilities of RXTE. Meanwhile, we must make the most of our current capabilities during the first few years of operation of GLAST. Ready, set, OBSERVE!!

\section{Acknowledgments}

The original work reported here was supported in part by National Science Foundation grant AST-0406865 and a number of NASA grants, most recently NNG05GM33G (RXTE), NNG05GO46G (RXTE), NNX08AJ64G (ADP), and Spitzer grant 1276552 (via JPL). The VLBA and VLA are instruments of the National Science Foundation operated by the National Radio Astronomy Observatory under cooperative agreement by Associated Universities Inc.

\section{References}

[1] J. Albert et al. Discovery of Very High Energy $\gamma$-ray Emission from the Low-Frequency Peaked BL Lac Object BL Lacertae, ApJ 666, L17-L20 (2007).

[2] M. Böttcher et al. The WEBT Campaign on the Blazar 3C 279 in 2006, ApJ 670, 968-977 (2007).

[3] B.J. Burn. On the Depolarization of Discrete Radio Sources by Faraday Dispersion, MNRAS 133, 67-83 (1966).

[4] R. Chatterjee et al. Correlated Multi-Waveband Variability in the Blazar 3C 279 from 1996 to 2007, $A p J$, in press (2008).

[5] R. Chatterjee et al. Association of Events in Radio, Optical, and X-ray Light Curves with Superluminal Knots in $3 C$ 120, in preparation (2009).

[6] F.D. D'Arcangelo et al. Rapid Multiwaveband Polarization Variability in the Quasar PKS0420-014: Optical Emission from the Compact Radio Jet ApJ, 659, L107-L110 (2007).

[7] R.P. Fender, T.M. Belloni, \& E. Gallo. Towards a Unified Model for Black Hole X-ray Binary Jets, MNRAS 355, 1105-1118 (2004).

[8] T.W. Jones. Polarization as a Probe of Magnetic Field and Plasma Properties of Compact Radio Sources - Simulation of Relativistic Jets, ApJ 332, 678-695 (1988).

[9] S.G. Jorstad et al. Polarimetric Observations of 15 Active Galactic Nuclei at High Frequencies: Jet Kinematics from Bimonthly Monitoring with the Very Long Baseline Array, AJ 130 1418-1466 (2005).

[10] S.G. Jorstad et al. Multiwaveband Polarimetric Observations of 15 Active Galactic Nuclei at High Frequencies: Search for Correlated Polarization Behavior, AJ 134 799-824 (2007).

[11] S.G. Jorstad et al. Spectral Energy Distributions and Variability of Blazars Observed with Spitzer (tentative title), in preparation (2008).

[12] M. Livio, J.E. Pringle, \& A.R. King. The Disk-Jet Connection in Microquasars and Active Galactic Nuclei, ApJ, 593 184-188.

[13] M. Lyutikov, V.I., Pariev, \& D.C. Gabuzda. Polarisation and structure of relativistic parsec-scale $A G N$ jets, MNRAS 360, 869-891 (2005). 
[14] A.P. Marscher. Relativistic Jets in AGN and their Relationship to the Central Engine, in proceedings of VI Microquasar Workshop: Microquasars and Beyond, ed. T. Belloni, PoS 033, $\underline{025}$ (2006).

[15] A.P. Marscher et al. Observational Evidence for the Accretion-Disk Origin for a Radio Jet in an Active Galaxy, Nature 417 625-627 (2002).

[16] A.P. Marscher et al. The Inner Jet of an Active Galactic Nucleus as Revealed by a Radio-to- $\gamma$-ray Outburst, Nature 452 966-969 (2008).

[17] A.P. Marscher et al. Association of Radio, Optical, and X-ray Events in $3 C 279$ (tentative title), in preparation (2009).

[18] A.P. Marscher \& W.K. Gear. Models for High-Frequency Radio Outbursts in Extragalactic Sources with Application to the Early 1983 Millimeter to Infrared Flare of 3C273, ApJ 298, 114-127 (1985).

[19] I.M. McHardy et al. Simultaneous X-Ray and Infrared Variability in the Quasar 3C 273, MNRAS 310, 571 (1999).

[20] T. Savolainen, K. Wiik, E. Valtaoja, S.G. Jorstad, \& A.P. Marscher. Connections between Millimetre Continuum Variations and VLBI Structure in 27 AGN, A\&A 394 851-861 (2002).

[21] N. Vlahakis. Disk-Jet Connection, in Blazar Variability Workshop II: Entering the GLAST Era, ed. H.R. Miller et al., Astron. Soc. Pacific Conf. Ser. 350, 169-177 (2006). 